\title{
CRNAs Stepping Over from the Operating Room: Working Together to Battle the Pandemic
}

With more than 10 million cases of COVID-19 to date, there have been more than a quarter of a million deaths in the United States due to the virus. ${ }^{1}$ The effects of COVID on the respiratory system make this pandemic significant for health care personnel in critical care specialties whose training is essential for ventilator-dependent patients. The resulting health care disaster has called for health care personnel to augment other providers, especially in critical care units.

This month's Editor's Choice is an interesting report of a study examining the comfort level of certified registered nurse anesthetists (CRNAs) with critical care ventilators while caring for patients with COVID-19. In response to the pandemic, many medical centers severely reduced or cancelled elective surgery cases, resulting in a decreased need for CRNAs in the operating room. At the same time, critical care units filled with patients with COVID-19 who required mechanical ventilation. CRNAs were often called upon to help manage the ventilator-dependent patients with COVID-19. Roberts and colleagues $^{2}$ reported that just-in-time training helped increase the confidence of CRNAs in managing mechanical ventilation for patients with COVID-19. Their findings are not surprising given the background, education, and experience of CRNAs.

The history of nurse anesthetists goes back at least to the Civil War. Certification occurred in 1956 when the American Association of Nurse Anesthetists (AANA) established a credentialing process. ${ }^{3}$ Currently, there are about 55,000 CRNAs in the United States. CRNAs practice in hospitals, ambulatory surgery centers, pain management settings, and office surgery settings. CRNAs are sometimes the sole anesthesia providers in rural hospitals and are routinely assigned to far-forward medical teams as the only anesthesia provider. They sometimes practice with a physician anesthesiologist and at other times independently. ${ }^{4}$ The Council on Accreditation of Nurse Anesthesia Educational Programs is now the sole accreditor of the 124 nurse anesthesia educational programs, mostly at the doctoral level. ${ }^{5,6}$ The only certification body for CRNAs

\footnotetext{
The authors have disclosed no conflicts of interest.

Correspondence: Paul N Austin PhD CRNA, 14311 Harvest Moon Rd, Boyds, MD 20841.E-mail: paulaustin5@gmail.com.
}

DOI: $10.4187 /$ respcare. 08763 is the National Board of Certification and Recertification of Nurse Anesthetists. ${ }^{7}$

\section{See the Original Study on Page 199}

Admission requirements to nurse anesthesia programs include licensure as a registered nurse in the United States or Puerto Rico and at least 1 year of critical care experience, but most applicants possess an average of 3 years of such experience. ${ }^{4}$ The definition of "critical care experience" includes caring for patients requiring mechanical ventilation. Programs may require applicants to be certified in critical care nursing. Student registered nurse anesthetists complete graduate courses in advanced physiology, health assessment, and pharmacology. Other courses examine the respiratory effects of anesthetics and adjuncts, and respiratory changes due to severe diseases such as COPD and ARDS. During clinical training, student registered nurse anesthetists gain a minimum of 2,000 clinical hours and perform at least 600 anesthetics, the majority being general anesthetics, usually requiring some form of mechanical ventilation. ${ }^{5}$ Airway management, along with expertise in neurological, cardiovascular, and respiratory systems, are the hallmarks of the nurse anesthesia profession.

Prior critical care experience, advanced education, and rich clinical experience prepare CRNAs to augment various critical care roles during this pandemic. This support has been termed "stepping over," where CRNAs apply their critical care knowledge to patients in critical care units, a subset of patients different than their normal surgical patients. ${ }^{8}$ The value of CRNAs is recognized by disaster planners. There are reports of CRNAs augmenting all critical care personnel, including nurses, physicians, and respiratory therapists. ${ }^{9}$ The need for CRNAs to assist with the care of patients with COVID-19 in the critical care unit depends on factors including critical care areas with the greatest shortage of providers, the number of patients and their severity, and the supply of CRNAs. The AANA offers guidance on CRNAs as advanced practice providers in critical care settings. ${ }^{10-12}$ Planners should place CRNAs in roles where they can practice to their full scope to maximize benefits.

Some of the other factors that planners and CRNAs must consider include skill sets, privileges, government directives or guidelines, regulatory requirements for the specific 


\section{EDITORIALS}

role, and malpractice insurance coverage. CRNAs may be held to a higher standard of care and practice than the respiratory therapist or critical care nurse. Assuming a nontraditional role such as a respiratory therapist does not separate the CRNA from their advanced practice background and education. $^{12}$

In the current study, Roberts and colleagues ${ }^{2}$ shared that CRNAs must train on the specifics of the critical care ventilator. The design, function, and operation of critical care ventilators are markedly different compared with ventilators that are a part of the anesthesia workstation. It is not surprising that the CRNAs needed training on the specifics of these devices, as well as on the policies and documentation requirements of wherever they provided support. Documentation methods, especially associated with an electronic medical record, are sometimes quite different from those used by the CRNA in the operating room, and training on their use may require significant time. Respiratory therapists may also work closely with CRNAs and other anesthesia providers when anesthesia workstations are used to ventilate patients with COVID-19.

Anesthesia workstations may be repurposed when there is an insufficient supply of critical care ventilators..$^{13}$ CRNAs continue to offer valuable support to critical care units by planning for the use of and operating anesthesia workstations to ventilate patients with COVID-19. Simply transporting the anesthesia workstation to the ICU to augment ventilation capacity belies the complexity and differences between these devices and critical care ventilators. ${ }^{14-16}$ Anesthesia workstations are significantly different in function and operation when compared to critical care ventilators. CRNAs are intimately familiar with anesthesia workstations, including the ventilators. CRNAs not only oversee their use but provide education on the basics of their operation. This is a potential added benefit of the CRNA in the ICU, serving as the expert to instruct the ICU staff on the nuances of ventilation with an anesthesia workstation.

The study by Roberts et $\mathrm{al}^{2}$ is foundational and informing. Future researchers should consider measuring the level of performance of CRNAs when collaborating with respiratory therapists as well as perceptions of the CRNAs. Additional future research is needed to expand the understanding of the role of CRNAs and other health care personnel during national health care emergencies. Examining critical care resources, including respiratory therapists and CRNAs, secondary to expanded surge capacity would assist future health care disaster preparedness. This is particularly vital due to the pandemic's volatile hot spot surges. How might future planning affect the critical care staffing needs for respiratory therapists and CRNAs in the ICU?

This pandemic requires all of us to be creative and to assume nontraditional roles. The importance of CRNAs collaborating with respiratory therapists to assist with critically ill patients with COVID-19 cannot be understated. CRNAs are not simply extenders; they remain a significant addition to the critical care team during this challenging time. Health care providers have put aside parochialism and tradition to collaborate in caring for the victims of this pandemic.

Paul N Austin

Graduate Programs of Nurse Anesthesia

Texas Wesleyan University

Fort Worth, Texas

Shari M Burns

Doctor of Nurse Anesthesia Practice Program

Midwestern University

Glendale, Arizona

\section{REFERENCES}

1. Centers for Disease Control and Prevention. CDC COVID data tracker. Available at: https://covid.cdc.gov/covid-data-tracker/\#cases_ casesper100klast7days. Accessed November 12, 2020.

2. Roberts KJ, Johnson B, Morgan MM, Vrontisis JM, Young KM, Czerpak E, et al. Evaluation of respiratory therapist extender comfort with mechanical ventilation during COVID-19. Respir Care 2021; 66:199-204.

3. Ray WT, Desai SP. The history of the nurse anesthesia profession. J Clin Anesth 2016;30:51-58.

4. American Association of Nurse Anesthetists. Certified registered nurse anesthetist fact sheet. Available at: https://www.aana.com/ membership/become-a-crna/crna-fact-sheet. Accessed November 17, 2020.

5. Council on Accreditation of Nurse Anesthesia Educational Programs. Standards for accreditation of nurse anesthesia educational programspractice doctorate. 2015; revised Oct 11, 2019. Available at: https:// www.coacrna.org/accreditation/accreditation-standards-policies-andprocedures-and-guidelines. Accessed November 17, 2020.

6. Council on Accreditation of Nurse Anesthesia Educational Programs. CRNA school search. Available at: https://www.coacrna.org/ programs-fellowships/crna-school-search. Accessed November 17, 2020.

7. National Board of Certification and Recertification of Nurse Anesthetists. For the public. Available at: https://www.nbcrna.com/ about-us/public. Accessed November 17, 2020.

8. National Academies of Sciences, Engineering, and Medicine. Rapid expert consultation on staffing considerations for crisis standards of care for the COVID-19 pandemic (July 28, 2020). Washington, DC: The National Academies Press; 2020. CrossRef.

9. Harris GH, Baldisseri MR, Reynolds BR, Orsino AS, Sackrowitz R, Bishop JM. Design for implementation of a system-level ICU pandemic surge staffing plan. Crit Care Explor 2020;2(6):e0136.

10. American Association of Nurse Anesthetists. Nurse anesthetists responding to COVID-19 in rural America. Available at: https://www. newswise.com/coronavirus/nurse-anesthetists-responding-to-covid19-in-rural-america/?article_id=731623. Accessed November 24, 2020.

11. American Association of Nurse Anesthetists. CRNAs as advanced practice providers in critical care settings. Available at: https://www. aana.com/docs/default-source/practice-aana-com-web-documents(all)/crnas-as-advanced-practice-providers-in-clinical-care-settings. pdf?sfvrsn=da009643_6. Accessed November 17, 2020. 


\section{EDITORIALS}

12. American Association of Nurse Anesthetists. CRNAs asked to assume critical care responsibilities during the COVID-19 pandemic. Available at: https://www.aana.com/docs/default-source/practiceaana-com-web-documents-(all)/crnas_asked_to_assume_critical_care_ responsibilities_during_the_covid_19_pandemic.pdf?sfvrsn=ea3630e7_ 6. Accessed November 17, 2020.

13. US Food and Drug Administration. Ventilator supply mitigation strategies: letter to health care providers. March 22, 2020. Available at: https://www.fda.gov/medical-devices/letters-health-care-providers/ ventilator-supply-mitigation-strategies-letter-health-care-providers. Accessed November 16, 2020.

14. Dosch M. Using the anesthesia workstation as a ventilator for critically ill patients: technical considerations. AANA J 2020;83(3 online content):13-17.

15. Haina KMK, Jr. Use of anesthesia machines in a critical care setting during the coronavirus disease 2019 pandemic. A A Pract 2020;14(7):e01243.

16. Austin PN, Dosch MP. Transporting anesthesia workstations to hospitals for use as ventilators. AANA J 2020;83(3 online content):24-28. 